\title{
Eliminating interference by anthocyanin in chlorophyll estimation of sweet potato (Ipomoea batatas L.) leaves
}

\author{
Wen-Dar Huang ${ }^{1}$, Kuan-Hung Lin ${ }^{2}$, Ming-Huang Hsu ${ }^{3}$, Meng-Yuan Huang ${ }^{4}$, Zhi-Wei Yang ${ }^{5}$, Pi-Yu Chao ${ }^{6 *}$ \\ and Chi-Ming Yang ${ }^{4^{*}}$
}

\begin{abstract}
Background: Spectral reflectance was evaluated for its usefulness as a nondestructive estimation of chlorophyll (Chl) content from three cultivars of sweet potato (Ipomoea batatas L.) with green, yellow, and purple leaves grown in a greenhouse for 22 days. While the green and yellow leaves contain variant amount of photosynthetic pigments without or with little level of anthocyanins, the purple leaves, except large amount of photosynthetic pigments, have high quantity of anthocyanins.

Results: For green and yellow leaves, the reciprocal reflectance $\left(\mathrm{R}^{-1}\right)$ and derived indices incorporating near infrared (NIR) reflectance, $\left[\left(R_{\lambda}\right)^{-1}-\left(R_{N I R}\right)^{-1}\right]$ and $\left[\left(R_{N I R} / R_{\lambda}\right)-1\right]$, in the green and red edge spectral ranges were shown to be strongly correlated $\left(r^{2}=0.8 \sim 0.9\right)$ with the chlorophyll content. The root mean square error (RMSE) of the chlorophyll content estimation using these indices was $<50 \mathrm{mg} \mathrm{m}^{-2}$. However, when purple leaves containing high levels of anthocyanins were included in the sample, $\mathrm{R}^{-1}$ in the green spectral range and the above-mentioned indices displayed much weaker correlations with the chlorophyll content. The RMSE of chlorophyll estimation using these indices in the green spectral range sharply increased to $>110 \mathrm{mg} \mathrm{m}^{-2}$ when the sample included purple leaves. The new index, $\left[1-\left(R_{\lambda} / R_{N I R}\right)\right]$, was therefore inferred and developed to eliminate the distorting effect of anthocyanins on chlorophyll content estimation using reflectance in the green spectral range. For leaves with high levels of anthocyanins, the correlation between $\left[1-\left(R_{\lambda} / R_{N N R}\right)\right]$ and the chlorophyll content remained strong $\left(r^{2}=0.8 \sim 0.9\right)$ in the green spectral range, and the RMSE was minimal.
\end{abstract}

Conclusion: The reflectance index, $\left[1-\left(R_{\lambda} / R_{N I R}\right)\right]$, therefore represents a new and useful parameter for estimating leaf chlorophyll content in leaves with any level of anthocyanins such as purple rice leaf.

Keywords: Sweet potato leaves; Chlorophyll; Anthocyanins; Spectral reflectance

\section{Multilingual abstracts}

Please see Additional file 1 for translations of the abstract into the six official working languages of the United Nations.

\section{Background}

The chlorophylls (Chls), Chl- $a$ and $-b$, are the most important plant pigments as they are necessary for

\footnotetext{
* Correspondence: pychao@faculty.pccu.edu.tw; cmyang@gate.sinica.edu.tw ${ }^{6}$ Department of Food, Health and Nutrition Science, Chinese Culture University, Xulin, Taipei, ROC 111, Taiwan

${ }^{4}$ Biodiversity Research Center, Academia Sinica, Nangang, Taipei, ROC 115, Taiwan

Full list of author information is available at the end of the article
}

photosynthesis. Chlorophylls absorb solar radiation and convert light energy to chemical energy. The amount of solar radiation that a leaf absorbs is determined mainly by chlorophylls. Thus, the foliar chlorophyll content directly affects the photosynthetic potential and primary production (Filella et al., 1995). Since chlorophyll contains much of the nitrogen content of a leaf, the chlorophyll content can provide an indirect estimation of a plant's nutrient status (Moran et al., 2000). The chlorophyll content changes throughout the different stages of plant development, and it universally decreases in situations of plant stress and during senescence (Merzlyak and Gitelson, 1995).

\section{实}


Traditional methods of measuring leaf chlorophyll content are destructive. They require leaf extraction with organic solvents and spectrophotometric or HPLC measurements in solution, and are considered time-consuming and expensive (Gitelson et al., 2006; Blackburn, 2007). However, much attention has been devoted to developing nondestructive leaf optical methods as alternative means to measure leaf chlorophyll contents. These methods include transmittance and reflectance spectroscopy for chlorophyll content measurement, and can be applied across spatial scales because of their simplicity, rapidity, and nondestructive nature (Chappelle et al., 1992; Buschmann and Nagel 1993; Main et al., 2011).

Many theoretical models based on leaf reflectance were developed to predict the leaf chlorophyll content, water content, and other variables associated with the vegetative structure (Inoue et al., 1993; Jacquemoud et al., 1996; Dawson et al., 1998). Relationships between reflectance in the visible region of the spectrum and the chlorophyll content are fundamentally nonlinear (Gitelson and Merzlyak, 1997). However, reciprocal reflectances $\left(R^{-1}\right)$ in the green and red edge spectral regions were found to be closely related to the chlorophyll content in leaves with a wide range of pigment compositions and contents (Gitelson and Merzlyak, 1994). Indices using these spectral bands were suggested for chlorophyll estimation in leaves of various plant species (Zou et al., 2011; Dillen et al., 2012; Huang et al., 2012). Gitelson et al. (2003) reported that the index, $\left(R_{\lambda}\right)^{-1}-\left(R_{N I R}\right)^{-1}$, was linearly proportional to the chlorophyll content in the wide spectral bands $525 \sim 555$ and $695 \sim 725 \mathrm{~nm}$. Moreover, a derived index incorporating near infrared (NIR) reflectance, $\left[\left(\mathrm{R}_{\lambda}\right)^{-1}-\left(\mathrm{R}_{\mathrm{NIR}}\right)^{-1}\right] \times \mathrm{R}_{\mathrm{NIR}}$, was also used to adjust for differences in leaf structure, and this algorithm gives highly accurate estimation of chlorophyll levels. Recently, a three-band conceptual model of remotely estimating total chlorophyll contents in leaves was developed and produced accurate estimation (Gitelson et al., 2006).

Leaf colors are determined by pigments, and anthocyanins are responsible for the red coloration in plant tissues (Ji et al., 1992; Gould et al., 1995). Therefore, leaves containing anthocyanins tend to be red or purple. Light in the spectral band at around $550 \mathrm{~nm}$ is absorbed by anthocyanins. The development of nondestructive methods for chlorophyll estimation using the green spectral band is more difficult for leaves containing anthocyanins, because their main absorption band is also located in the green range (Merzlyak et al., 2003). In anthocyanin-free leaves, nondestructive chlorophyll estimation are possible using either the green band (around $550 \mathrm{~nm}$ ), red edge band (around $700 \mathrm{~nm}$ ), or an NIR band of $>750 \mathrm{~nm}$. For leaves containing anthocyanins, use of only the red edge band or an NIR band at $>750 \mathrm{~nm}$ is recommended (Gitelson et al., 2006).
In applying the above developed models for nondestructively estimating chlorophyll contents in leaves with or without anthocyanins (Gitelson et al., 2006), big error was found in anthocyanin-containing leaves, suggesting that anthocyanins may affect the nondestructive estimation of chlorophyll content in all purple leaves. Actually, the same phenomenon was also found and bothered the authors for more than fifteen years in biochemical and destructive measurement of chlorophyll in leaves with any level of anthocyanins; in short, the higher content the anthocyanins, the bigger error the chlorophyll measurement (personal data not published). We referred that the anthocyanins strongly absorbing around 500$550 \mathrm{~nm}$ may strongly affect the chlorophyll-related compounds, including all chlorophyll biosynthesis and degradation intermediates, strongly absorbing 400$475 \mathrm{~nm}$ and 550-700 $\mathrm{nm}$. The more concentration of anthocyanins lead to the more distortion effect on chlorophyll measurement.

Therefore, the objectives of this study were to investigate the properties of the reflectance spectra of sweet potato leaves with and without anthocyanins, and to develop techniques for nondestructive chlorophyll estimation in leaves with a wide range of pigment contents. A technique was developed to eliminate the effects of anthocyanin on reflectance in the green spectral range on chlorophyll estimation, and a new index for estimating chlorophyll contents is finally proposed.

\section{Methods}

\section{Plant materials and culture practice}

Three leafy vegetable sweet potato [Ipomoea batatas (L.) Lam] cultivars of Taoyuan 2, green, yellow, and purple were used in this study. The green leaf cultivar was selected by the Taoyuan District Agricultural Research and Extension Station (TDARES) for food consumption of its leaves. Yellow and purple cultivars are local varieties from Taoyuan, and contain high amounts of carotenoids. Terminals about $40 \mathrm{~cm}$ in length were taken from sturdy vines, and cultivated in plastic boxes $60 \mathrm{~cm}$ long, $22 \mathrm{~cm}$ wide, and $15 \mathrm{~cm}$ deep, that contained a medium of sand, vermiculite, and loamy soil in a volume ratio of 2:1:1. Specimens were planted in a greenhouse at the TDARES, and evenly spaced at intervals of $60 \mathrm{~cm}$ to encourage similar growth rates and sizes. Plants were treated with the nutrients $\mathrm{N}: \mathrm{P}_{2} \mathrm{O}_{5}: \mathrm{K}_{2} \mathrm{O}$ (0.1:0.2:0.3 g/box) weekly, watered every other day to maintain optimal irrigation, and allowed to grow for $22 \mathrm{~d}$ before the following measurements of spectral reflectance were made.

\section{Measurement of spectral reflectance}

Adaxial reflectance spectra of leaves were recorded at a rate of $600 \mathrm{~nm} / \mathrm{min}$ using an U-3010 spectrophotometer (Hitachi, Tokyo, Japan) equipped with an integrating 
sphere attachment. The diffuse reflectance of the leaves was measured at values of $400 \sim 800 \mathrm{~nm}\left(\mathrm{R}_{400} \sim \mathrm{R}_{800}\right)$ with a spectral resolution of $1 \mathrm{~nm}$ and against barium sulfate as a reference standard. The reflectance was expressed as a ratio of the radiance of the leaf to the radiance of the reference. The spectra were recorded for sections of leaves between the main veins.

\section{Pigment determinations}

Chlorophylls and carotenoids were isolated from leaves by homogenization in liquid nitrogen and subsequent threefold extraction with $80 \%$ acetone $(\mathrm{v} / \mathrm{v})$. After centrifugation for $5 \mathrm{~min}$ at $1500 \times g$, the absorbance of the supernatant was measured at 663.6, 646.6, and $440.5 \mathrm{~nm}$ (Porra et al., 1989). Leaf samples were also extracted with $1 \%(\mathrm{w} / \mathrm{v}) \mathrm{HCl}$ in methanol, and the anthocyanin contents were assayed spectrophotometrically. The relative amounts of anthocyanins were expressed by $\left[\mathrm{A}_{530}-0.333 \mathrm{~A}_{657}\right] \mathrm{m}^{-2}$ (Mancinelli et al., 1975). Absorbance was measured with a Hitachi U-2000 UV-visible spectrophotometer.

\section{Statistical analysis}

To develop model datasets, 48 leaves from the three varieties were used. Validation was done using independent datasets derived from 24 leaves, and the chlorophyll content was calculated using reflectance data from the model's validation datasets. The predicted chlorophyll content was compared to chlorophyll levels physically measured, and the root mean square error (RMSE) of the predictions was calculated. Relationships between the reflectance indices and chlorophyll contents were examined using simple linear regression models. All statistical analyses were conducted using JMP software, vers. 5.01 (SAS Institute, Cary, NC, USA).

\section{Results}

\section{Pigment contents}

Variations in pigment contents of green, yellow, and purple leaves were observed and are given in Table 1 .
The chlorophyll content varied from $99 \mathrm{mg} \mathrm{m}^{-2}$ in yellow leaves to $615 \mathrm{mg} \mathrm{m}^{-2}$ in purple leaves. Purple leaves had the highest chlorophyll content, green leaves had an intermediate level, and yellow leaves had the lowest amount. The mean chlorophyll content in yellow $\left(132 \mathrm{mg} \mathrm{m}^{-2}\right)$ leaves was about $2 \sim 3$-times lower than that of green $\left(408 \mathrm{mg} \mathrm{m}^{-2}\right)$ and purple $\left(478 \mathrm{mg} \mathrm{m}^{-2}\right)$ leaves. While green and yellow leaves were found to be free of anthocyanins, purple leaves contained much of them.

\section{Reciprocal reflectance}

The reciprocal reflectance spectra $\left(\mathrm{R}^{-1}\right)$ in the blue wavelength ranging $400 \sim 500 \mathrm{~nm}$ was highest in purple leaves, but lower in green and yellow leaves (Figure 1). Values of $\mathrm{R}^{-1}$ were affected by absorption by both chlorophyll and carotenoids in this range. There were differences in $\mathrm{R}^{-1}$ values of green and yellow leaves in the $500 \sim 700-\mathrm{nm}$ range, with yellow leaves being lower. A blue-shift accompanied a decrease in chlorophyll content at both the green edge (around $530 \mathrm{~nm}$ ) and red edge (around $700 \mathrm{~nm}$ ). In purple leaves, the change in $\mathrm{R}^{-1}$ values was not notable in this range. Purple leaves, which had the highest chlorophyll content, also had the highest $\mathrm{R}^{-1}$; conversely yellow leaves, with the lowest chlorophyll content, also had the lowest $\mathrm{R}^{-1}$ (Table 1, Figure 1).

\section{Correlations of reflectance indices with the chlorophyll content, and the RMSE with chlorophyll estimates}

To investigate spectral bands where $\mathrm{R}^{-1}$ was sensitive to the chlorophyll content, coefficients $\left(r^{2}\right)$ of $\mathrm{R}^{-1}$ with the chlorophyll content and the RMSE with reflectance estimates of the chlorophyll content were examined. The model development data were divided into two sets. Because we focused on the anthocyanin contents of purple leaves, one set included only green and yellow leaves, while the other included purple leaves. For green and yellow $(\mathrm{G}+\mathrm{Y})$ leaves, $r^{2}$ was minimal in the blue $(400 \sim$ $500 \mathrm{~nm}$ ) and red (around $680 \mathrm{~nm}$ ) ranges. A consistently high level of correlation $\left(r^{2}=0.8 \sim 0.9\right)$ was observed

Table 1 The content of chlorophylls (Chl), carotenoids, and anthocyanins in leaves of three varieties of sweet potato

\begin{tabular}{|c|c|c|c|c|c|}
\hline \multirow[t]{2}{*}{ Data set } & \multirow[t]{2}{*}{$n$} & \multicolumn{2}{|c|}{$\mathrm{Chl}\left(\mathrm{mg} \mathrm{m}^{-2}\right)$} & \multirow[t]{2}{*}{ Carotenoids $\left(\mathrm{mg} \mathrm{m}^{-2}\right)$} & \multirow[t]{2}{*}{ Anthocyanins $\left(A_{530^{-}}-0.333 A_{657}\right) \mathrm{m}^{-2}$} \\
\hline & & Total & Mean & & \\
\hline \multicolumn{6}{|c|}{ Model development } \\
\hline Green & 16 & $319 \sim 515$ & 408 & $74 \sim 88$ & 0 \\
\hline Yellow & 16 & $99 \sim 182$ & 132 & $36 \sim 51$ & 0 \\
\hline Purple & 16 & $336 \sim 615$ & 478 & $73 \sim 88$ & $0.43 \sim 2.90$ \\
\hline \multicolumn{6}{|c|}{ Model validation } \\
\hline Green & 8 & $237 \sim 431$ & & $68 \sim 83$ & 0 \\
\hline Yellow & 8 & $104 \sim 145$ & & $33 \sim 47$ & 0 \\
\hline Purple & 8 & $297 \sim 545$ & & $74 \sim 89$ & $0.35 \sim 2.71$ \\
\hline
\end{tabular}




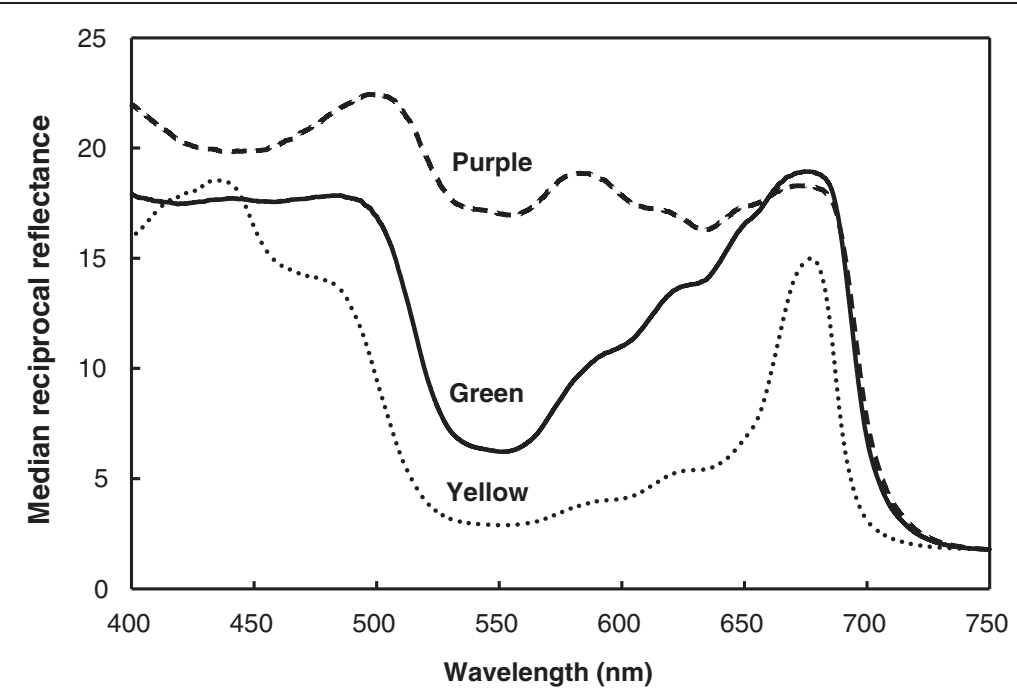

Figure 1 Reciprocal reflectance spectra for leaves of three varieties of sweet potato.
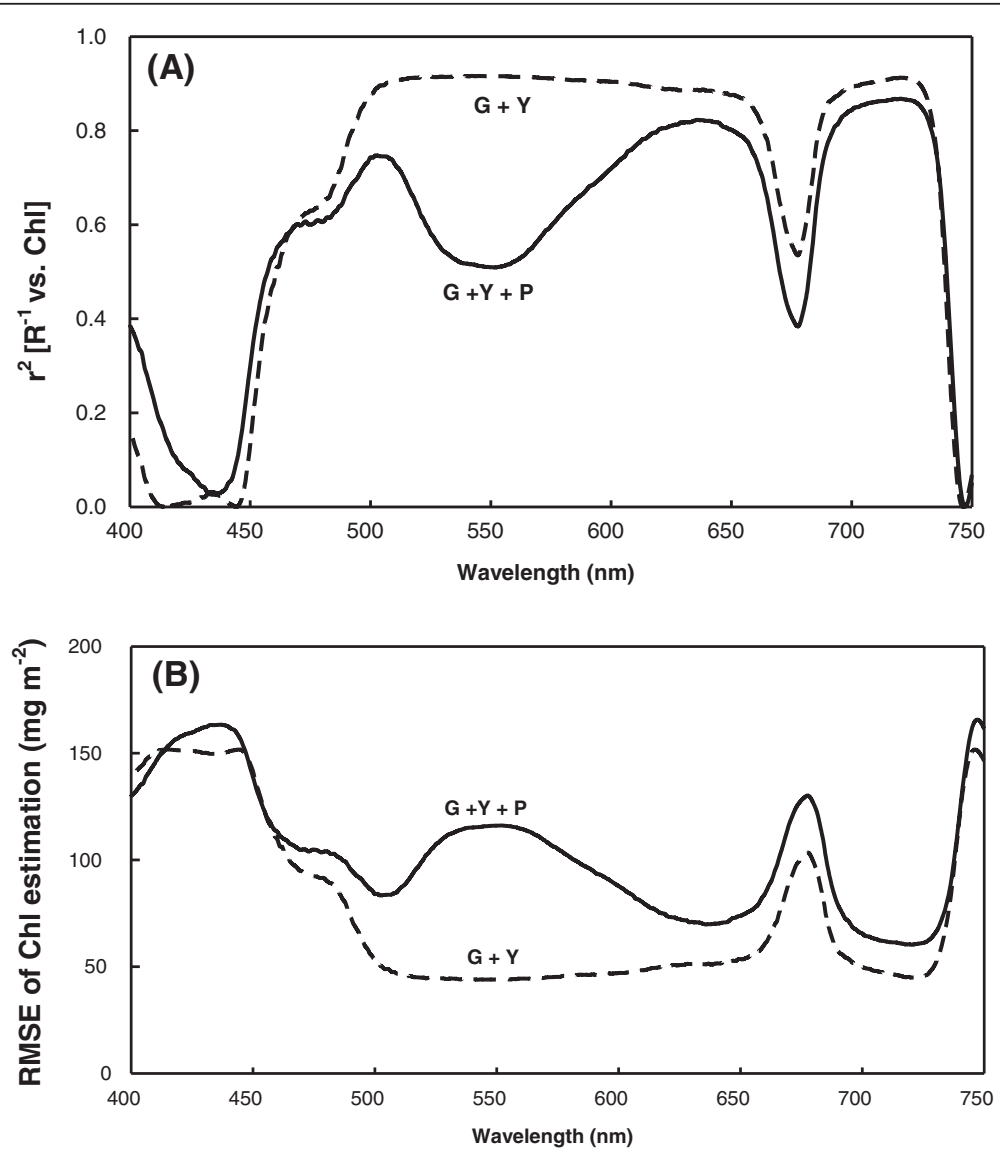

Figure 2 Coefficient between reciprocal reflectance and chlorophyll content and its RMSE. (A) Coefficient between reciprocal reflectance $\left(\mathrm{R}^{-1}\right)$ and chlorophyll content; (B) root mean square error (RMSE) of the chlorophyll estimation by $\mathrm{R}^{-1}$. $\mathrm{G}+\mathrm{Y}$, green and yellow leaves only; $\mathrm{G}+\mathrm{Y}+\mathrm{P}$ : green, yellow, and purple leaves. 
over a broad range of $500 \sim 650 \mathrm{~nm}$ (green-red region of the spectrum) and near $700 \mathrm{~nm}$ (red edge) (Figure 2A). Compared to the other set, the set including purple leaves $(\mathrm{G}+\mathrm{Y}+\mathrm{P})$ had a significantly lower $r^{2}$ (of 0.51 ) between $\mathrm{R}^{-1}$ and the chlorophyll content in the green region of the spectrum at $500 \sim 600 \mathrm{~nm}$ (Figure 2A). In addition, the correlation was strong for all leaves $(G+Y+P)$ in the red region at around $640 \mathrm{~nm}$ and at the red edge at around $700 \mathrm{~nm}$. This demonstrates the applicability of $\mathrm{R}^{-1}$ as a method of measuring the total chlorophyll content. In these spectral ranges, the RMSE for chlorophyll estimation was minimal. The RMSE was $<50 \mathrm{mg} \mathrm{m}^{-2}$ for the green and yellow $(G+Y)$ set, and $>70 \mathrm{mg} \mathrm{m}^{-2}$ for the set with all $(G+Y+P)$ leaves (Figure 2B).

Two indices derived from the reciprocal reflectance, $\left[\left(R_{\lambda}\right)^{-1}-\left(R_{N I R}\right)^{-1}\right]$ and $\left[\left(R_{N I R} / R_{\lambda}\right)-1\right]$, were correlated to
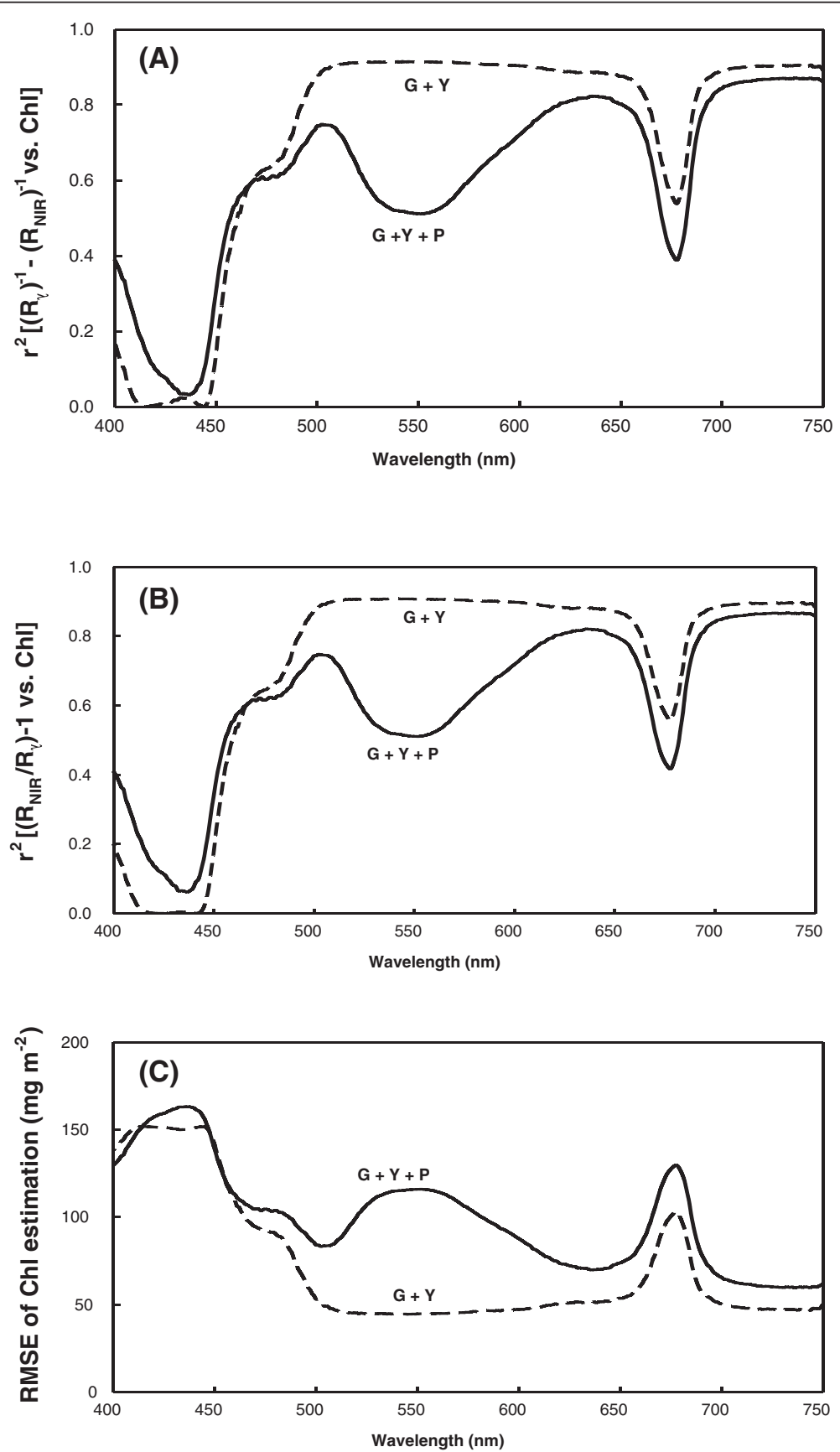

Figure 3 Coefficients between $\left[\left(R_{\lambda}\right)^{-1}-\left(R_{N I R}\right)^{-1}\right]$ and $\left[\left(R_{N I R} / R_{\lambda}\right)-1\right]$ and the chlorophyll content and their RMSE. (A) Coefficient between $\left[\left(R_{\lambda}\right)^{-1}-\left(R_{N I R}\right)^{-1}\right]$ and the chlorophyll content; $(B)$ coefficient between $\left[\left(R_{N I R} / R_{\lambda}\right)-1\right]$ and the chlorophyll content; (C) root mean square error (RMSE) of chlorophyll estimation by $\mathrm{R}^{-1}$. 
the total chlorophyll content in wide spectral ranges. Coefficients of $\left[\left(R_{\lambda}\right)^{-1}-\left(R_{N I R}\right)^{-1}\right]$ vs. chlorophyll (Figure 3A) and $\left[\left(R_{N I R} / R_{\lambda}\right)-1\right]$ vs. chlorophyll (Figure $3 B$ ) presented a broad flat maximum in the respective spectral ranges of $500 \sim 650$ and $700 \sim 740 \mathrm{~nm}$ for green and yellow $(\mathrm{G}+\mathrm{Y})$ leaves, while $R_{N I R}$ is the reflectance in the NIR range of $750 \sim 800 \mathrm{~nm}$. In these spectral ranges for $\mathrm{G}+\mathrm{Y}$ leaves, the RMSE of the chlorophyll estimation by these indices was $<50 \mathrm{mg} \mathrm{m}^{-2}$ (Figure 3C). When using all leaves, including purple $(\mathrm{G}+\mathrm{Y}+\mathrm{P})$, as the dataset, $r^{2}$ decreased down to $<0.6$ in the green region of the spectrum at 500 $\sim 600 \mathrm{~nm}$ (Figure 3A, B), but the RMSE increased to $>110 \mathrm{mg} \mathrm{m}^{-2}$ (Figure 3C). It is obvious that anthocyanins affect the chlorophyll estimation, and it seems that no existed models can eliminate this distortion effect.

To eliminate the effect of anthocyanins on the reflectance in the green spectral range, we developed a new index, $\left[\left(\mathrm{R}_{\lambda}\right)^{-1}-\left(\mathrm{R}_{\mathrm{NIR}}\right)^{-1}\right] \times \mathrm{R}_{\lambda}=1-\left(\mathrm{R}_{\lambda} / \mathrm{R}_{\mathrm{NIR}}\right)=\left(\mathrm{R}_{\mathrm{NIR}}-\mathrm{R}_{\lambda}\right) /$ $\mathrm{R}_{\mathrm{NIR}}$, derived from $\left[\left(\mathrm{R}_{\lambda}\right)^{-1}-\left(\mathrm{R}_{\mathrm{NIR}}\right)^{-1}\right]$ and $\mathrm{R}_{\lambda}$, by just changing multiplying $\mathrm{R}_{\mathrm{NIR}}$ in denominato of Gileson's model (2006), $\left[\left(R_{\lambda}\right)^{-1}-\left(R_{N I R}\right)^{-1}\right] \times R_{N I R}=\left(R_{\lambda} / R_{N I R}\right)-1=\left(R_{N I R}-\right.$
$\left.R_{\lambda}\right) / R_{\lambda}$, with multiplying $R_{\lambda}$ in the new equation. The expectation for the new model is that $\left(R_{N I R}-R_{\lambda}\right) / R_{N I R}$, not only adjusts the difference for leaf structure, position, angle and photosynthetic pigments, but also narrows down the reciprocal spectral difference between the anthocyanin-free leaves (the less amount of anthocyanins) and the anthocyanins-containing leaves (the most amount of anthocyanins), and therefore maximally decreases the influence of anthocyanins on the chlorophyll estimation, whereas the old model $\left(R_{N I R}-R_{\lambda}\right) / R_{\lambda}$ broaden the difference and therefore maximally increases the influence. In the spectral ranges of $500 \sim 650$ and $700 \sim 750 \mathrm{~nm}$, the new function $1-\left(R_{\lambda} / R_{N I R}\right)$ vs. chlorophyll was linear for green and yellow $(G+Y)$ leaves (Figure $4 A)$. As with the indices above, for this index $r^{2}=0.9$, and the RMSE was still $<50 \mathrm{mg} \mathrm{m}^{-2}$ (Figure 4B). Unlike the previous indices, when this index was applied to the full set of leaves $(\mathrm{G}+\mathrm{Y}+\mathrm{P})$, including the anthocyanin-containing purple leaves, there was no significant decrease in $r^{2}$ in the green region of the spectrum at $500 \sim 600 \mathrm{~nm}$. The present RMSE also remained low at $<70 \mathrm{mg} \mathrm{m}^{-2}$.
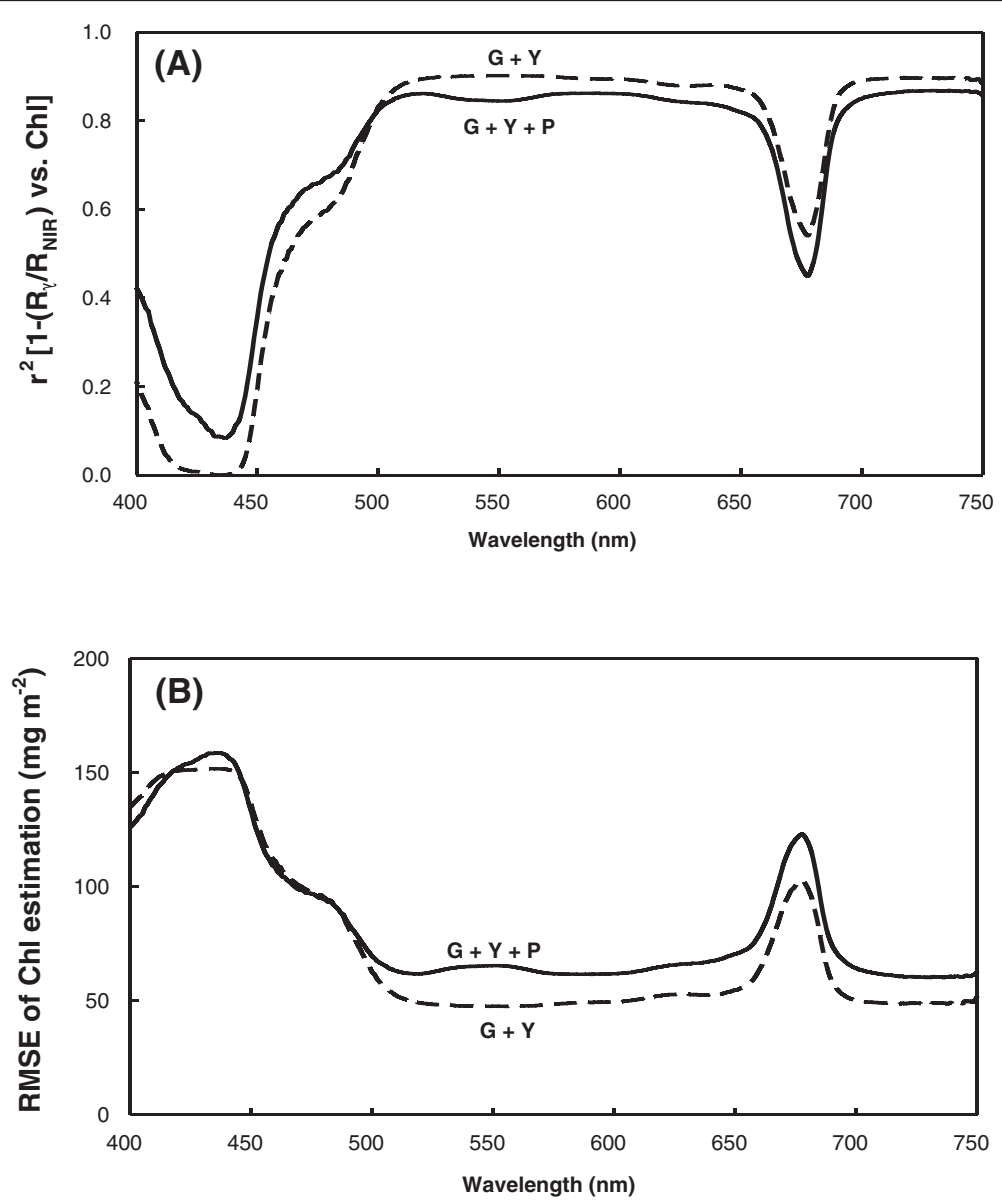

Figure 4 Coefficient between [1 - $\left.\left(\mathbf{R}_{\lambda} / \mathbf{R}_{\text {NIR }}\right)\right]$ and the chlorophyll content and its RMSE. (A) Coefficient between $\left[1-\left(R_{\lambda} / R_{N I R}\right)\right]$ and the chlorophyll content; (B) root mean square error (RMSE) of the chlorophyll estimation by $\mathrm{R}^{-1}$. 
The main difference in the behavior of these two indices, $\left(R_{N I R} / R_{\lambda}\right)-1$ (Figure 3$)$ and $1-\left(R_{\lambda} / R_{N I R}\right)$ (Figure 4$)$, was in the green range $(500 \sim 600 \mathrm{~nm})$ for anthocyanincontaining leaves (Figure 5). The coefficient of $\left(R_{N I R} / R_{\lambda}\right)$ 1 vs. the chlorophyll content exhibited a notable decrease down to around 0.55 in the green range (Figure 5A), and the RMSE of the chlorophyll estimation using the index $\left(R_{N I R} / R_{\lambda}\right)-1$ rose to $110 \mathrm{mg} \mathrm{m}^{-2}$ in the green range (Figure $5 \mathrm{~B})$. In contrast, the $r^{2}$ of $1-\left(\mathrm{R}_{\lambda} / \mathrm{R}_{\mathrm{NIR}}\right)$ vs. the chlorophyll content remained around $0.8 \sim 0.9$ in the green range, and the RMSE of the chlorophyll estimation by the index $1-\left(R_{\lambda} / R_{N I R}\right)$ was also still minimal, remaining at $<70 \mathrm{mg} \mathrm{m}^{-2}$.

\section{Correlations between the predicted and actual measured chlorophyll contents}

To validate the proposed model, the new index, $1-\left(R_{\lambda} /\right.$ $R_{N I R}$ ), was used to estimate the chlorophyll content in independent datasets with widely varying pigment contents (Table 1). The chlorophyll content was predicted using the reflectance data from the validation datasets, the predictions were compared to actual measured chlorophyll values, and the RMSE of the chlorophyll prediction was determined. Correlations between the predicted and measured chlorophyll contents were $r^{2}=$ 0.94 for $1-\left(R_{550} / R_{N I R}\right)$ (Figure $6 \mathrm{~A}$ ), and $r^{2}=0.93$ for 1 $\left(\mathrm{R}_{705} / \mathrm{R}_{\mathrm{NIR}}\right)$ (Figure $\left.6 \mathrm{~B}\right)$. The RMSEs of the chlorophyll predictions for $R_{550}$ and $R_{705}$ were $<38$ and $<43 \mathrm{mg} \mathrm{m}^{-2}$, respectively.

Rather than the usual narrow band channel, reflectances in the wide band ranges of $550 \sim 590$ and $700 \sim$ $740 \mathrm{~nm}$ can also be used as the terms in the index, $1-\left(\mathrm{R}_{\lambda} / \mathrm{R}_{\mathrm{NIR}}\right)$. For the $550 \sim 590$ - and $700 \sim 740-\mathrm{nm}$ ranges, correlations between the predicted and measured chlorophyll contents were $r^{2}=0.94$ for both $1-\left(R_{550 ~ 590} /\right.$ $\mathrm{R}_{\text {NIR }}$ ) (Figure $7 \mathrm{~A}$ ) and $1-\left(\mathrm{R}_{700 \sim 740} / \mathrm{R}_{\text {NIR }}\right)$ (Figure $7 \mathrm{~B}$ ), with RMSEs of $<38.5$ and $38.9 \mathrm{mg} \mathrm{cm}^{-2}$, respectively.

\section{Discussion}

It was previously shown that the blue and red spectral ranges, the main absorption bands of chlorophyll, are only sensitive to low chlorophyll levels, but at moderate
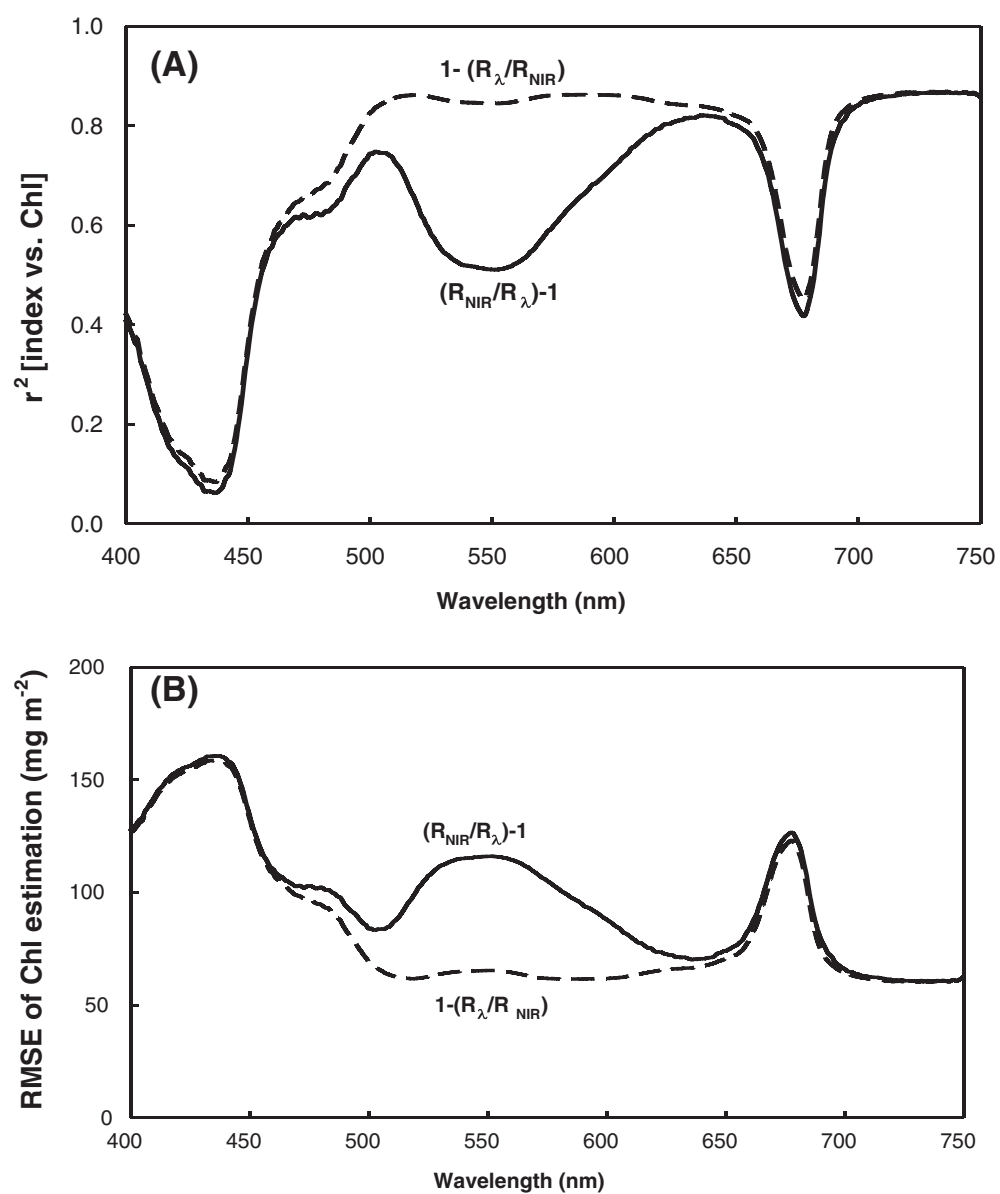

Figure 5 Coefficients of $\left[\left(R_{N I R} / R_{\lambda}\right)-1\right]$ and [1 $\left.-\left(R_{\lambda} / R_{N I R}\right)\right]$ with the chlorophyll content of all leaves and their RMSEs. (A) Coefficients of $\left[\left(R_{N I R} / R_{\lambda}\right)-1\right]$ and $\left[1-\left(R_{\lambda} / R_{N I R}\right)\right]$ with the chlorophyll content of all leaves $(G+Y+P)$; $(B)$ root mean square error (RMSE) of the chlorophyll estimation by $\mathrm{R}^{-1}$. 


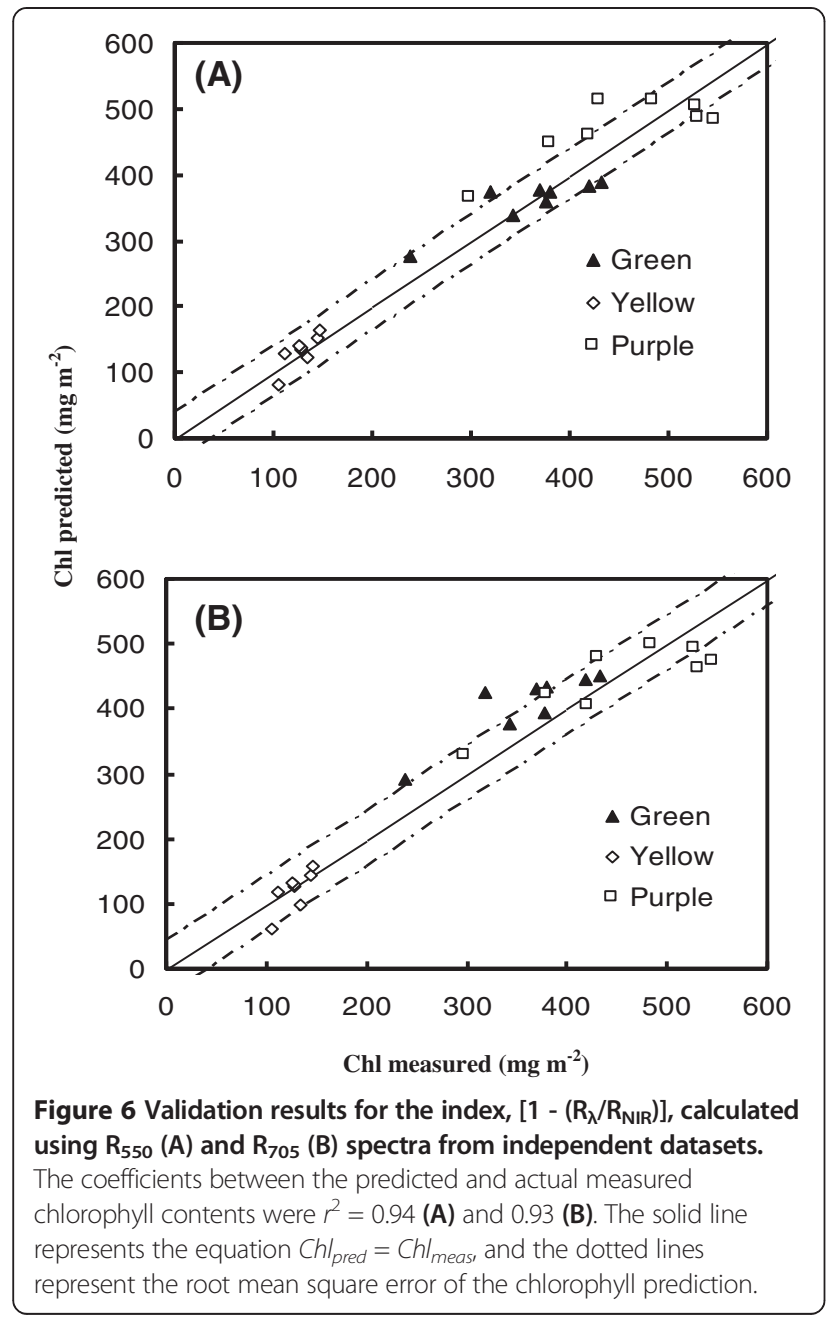

to high levels, they become insensitive (Gitelson and Merzlyak, 1994, 1996, 1997). In these spectral ranges, reflectance is only sensitive to total chlorophyll levels at $90 \mathrm{mg} \mathrm{m}^{-2}$ (Gitelson et al., 2003). Such low levels of chlorophyll are found in highly stressed or senescent leaves. The three sweet potato varieties used in this study grew well under greenhouse conditions, and the lowest chlorophyll content recorded was $99 \mathrm{mg} \mathrm{m}^{-2}$ in yellow leaves (Table 1). In both the model development and validation datasets, chlorophyll contents were not low. Therefore, the blue and red spectral reflectances for sweet potato leaves should be insensitive to chlorophyll content. For the green and yellow $(\mathrm{G}+\mathrm{Y})$ sweet potato leaves, $R_{\lambda}$ values in the visible blue $(400 \sim 500 \mathrm{~nm})$ and visible red $(660 \sim 690)$ ranges showed the lowest correlations with chlorophyll content, but $R_{\lambda}$ values showed maximum sensitivity to the chlorophyll content in the green $(500 \sim 600 \mathrm{~nm})$ and red edge (around $700 \mathrm{~nm}$ ) ranges (Figure 2A). This was due to the strong absorption by chlorophyll in the blue $(400 \sim 500 \mathrm{~nm})$ and red $(660 \sim 690 \mathrm{~nm})$ spectral ranges, but weak absorption in

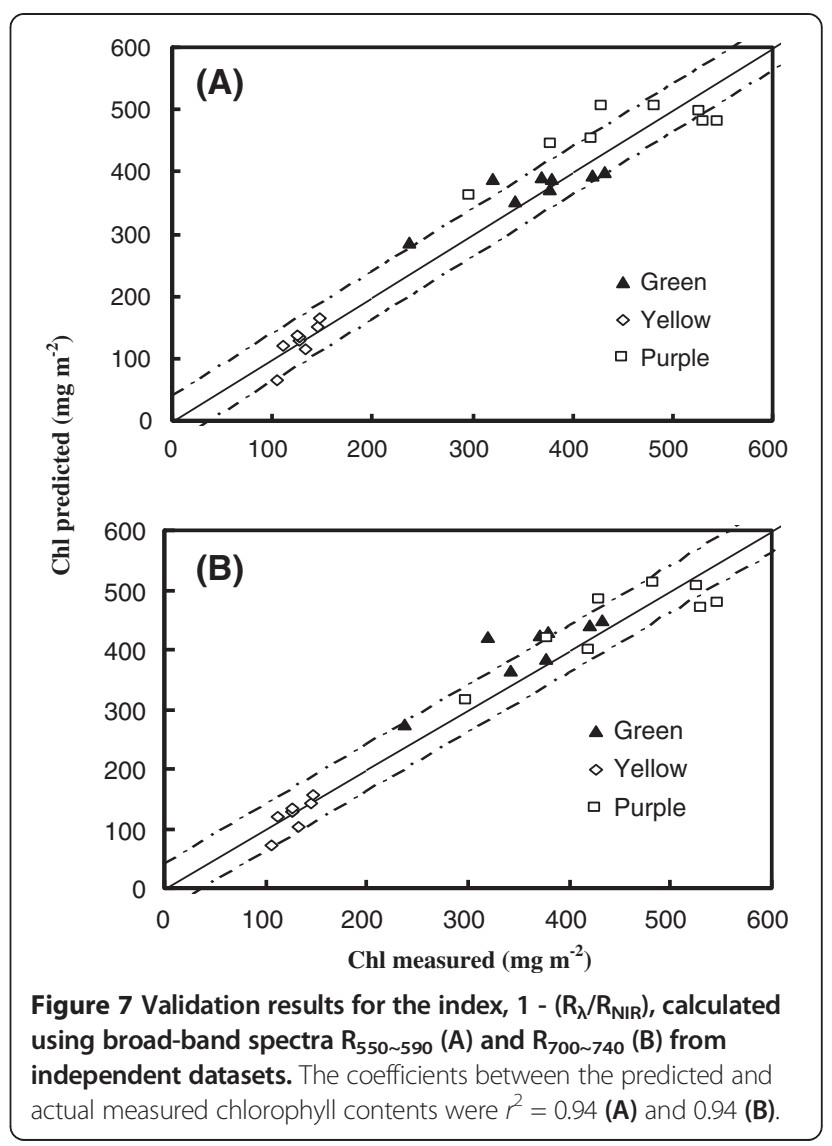

the green $(500 \sim 600 \mathrm{~nm})$ and red edge areas (around $700 \mathrm{~nm}$ ). Therefore, reflectance in the green and red edge ranges can be used as more-precise measurements for developing leaf chlorophyll content estimation algorithms (Lichtenthaler et al., 1996; Gamon and Surfus, 1999; Gitelson et al., 2002). For green and yellow $(G+Y)$ leaves, three indices derived from the reciprocal reflectance, $\left[\left(R_{\lambda}\right)^{-1}-\left(R_{N I R}\right)^{-1}\right],\left[\left(R_{N I R} / R_{\lambda}\right)-1\right]$, and [1 - $\left.\left(R_{\lambda} / R_{N I R}\right)\right]$, displayed strong correlation with the total chlorophyll content in the green and red edge spectral ranges (Figures 3A, B, 4A).

The relationship between the spectral reflectance and chlorophyll content may be influenced by the presence of other pigments. Curran et al. (1991) found that the red edge was significantly shifted to longer wavelengths by amaranthin, a red pigment found in Amaranthus tricolor leaves. In anthocyanin-containing leaves, both chlorophyll and anthocyanin contribute to absorption and reflectance in the green spectral range. The development of a nondestructive method for estimating chlorophyll content is complicated by the interference of absorption by anthocyanins in leaves and fruits which contain them (Gitelson et al., 2001). In anthocyanin-free leaves and fruits, reflectances at the green $(550 \mathrm{~nm})$ and red edges (around $700 \mathrm{~nm}$ ) were closely correlated with 
a wide range of chlorophyll contents (Gitelson et al., 2003). In leaves and fruits containing anthocyanins, a strong correlation was observed between the chlorophyll content and $R_{550}$, while $R_{700}$ was lost due to anthocyanin absorption at $550 \mathrm{~nm}$ causing the reflectance to be significantly lower than at $700 \mathrm{~nm}$. This meant that spectral bands at around $550 \mathrm{~nm}$ were seen as unsuitable for nondestructive chlorophyll estimation in anthocyanincontaining leaves (Gitelson et al., 2001). In this study, only purple leaves contained anthocyanins (Table 1); therefore, correlations between the old derived indices of $\left[\left(R_{\lambda}\right)^{-1}-\left(R_{N I R}\right)^{-1}\right]$ and $\left[\left(R_{N I R} / R_{\lambda}\right)-1\right]$ and chlorophyll content were relatively weak in the green spectral range when the dataset included purple leaves. The RMSE of chlorophyll estimates using $\mathrm{R}^{-1}$ in the green spectral range was dramatically increased by the inclusion of anthocyanin-containing leaves. Independent of the presence of anthocyanins, $\mathrm{R}^{-1}$ indices were found to be highly sensitive to the total chlorophyll content in the red edge spectral range (Figures 2, 3).

When developing indices for nondestructive chlorophyll estimation, spectral bands at around $550 \mathrm{~nm}$ are not recommended for leaves which contain anthocyanins (Gitelson et al., 2003). Hence, we attempted to determine whether spectral reflectance in the green range could be used as a sensitive term in developing algorithms for estimating the chlorophyll content of leaves containing anthocyanins. The new index, $\left[1-\left(R_{\lambda} / R_{N I R}\right)\right]$, was therefore developed to eliminate the effect of anthocyanins on reflectance in the green spectral range. Using this new index to predict the chlorophyll content, no sharp decrease in $r^{2}$ or increase in RMSE was observed following the inclusion of purple leaves in the dataset (Figures 4,5 ). This means that the green range reflectance becomes a usable term for estimating the chlorophyll content in anthocyanin-containing leaves.

With validation of the indices, $\left[1-\left(R_{550} / R_{N I R}\right)\right]$ and [1 $\left(R_{705} / R_{\text {NIR }}\right)$ ], they are shown to be accurate predictors of the chlorophyll content of leaves containing anthocyanins (Figure 6). To significantly increase the sensitivity and signal-to-noise ratio and decrease the cost of reflectometers, the use of wide-band filters is allowed in reflectometers (Gitelson et al., 2003). Reflectances in the wide band ranges of $550 \sim 590$ and $700 \sim 740 \mathrm{~nm}$ were used as parameters in the index, $1-\left(\mathrm{R}_{\lambda} / \mathrm{R}_{\mathrm{NIR}}\right): 1-\left(\mathrm{R}_{550 \sim 590} / \mathrm{R}_{\mathrm{NIR}}\right)$ and $1-\left(R_{700 \sim 740} / R_{\text {NIR }}\right)$. Validation demonstrated that the chlorophyll content could be accurately estimated for leaves which contained anthocyanins (Figure 7). Therefore, independent of the presence of anthocyanins, spectral bands in the green or red edge ranges are sufficient for nondestructive chlorophyll estimation when used as parameters in the index, $1-\left(\mathrm{R}_{\lambda} / \mathrm{R}_{\mathrm{NIR}}\right)$. This new index, 1 - $\left(R_{\lambda} / R_{\text {NIR }}\right)$, and old index, $\left(R_{\text {NIR }} / R_{\lambda}\right)-1$, have been applied to nondestructively assess the chlorophyll content of yellow, green and purple rice cultivars; the coefficients between the predicted and actual measured chlorophyll contents were $r^{2}=0.91$ and 0.92 , respectively (Figure 8). Therefore, it is expected that the present new index could be comprehensively used to nondestructively estimate the plant leaves containing anthocyanins with high $r^{2}$ and low RMSE, while other indices in the literature are not.

\section{Conclusions}

In previous studies, spectral bands in green were determined to be unsuitable for chlorophyll nondestructive estimation with leaves containing anthocyanins (Gitelson et al., 2006; Merzlyak et al., 2003). In the present study, we developed a new index to eliminate the effect of anthocyanins on the reflectance in the green range when estimating chlorophyll contents of leaves containing anthocyanins. We concluded that the index, $\left[1-\left(R_{\lambda} / R_{N I R}\right)\right]$, used with reflectance in the green and red edge ranges, is sufficient for nondestructive chlorophyll estimation in spite of the presence or absence of anthocyanins.

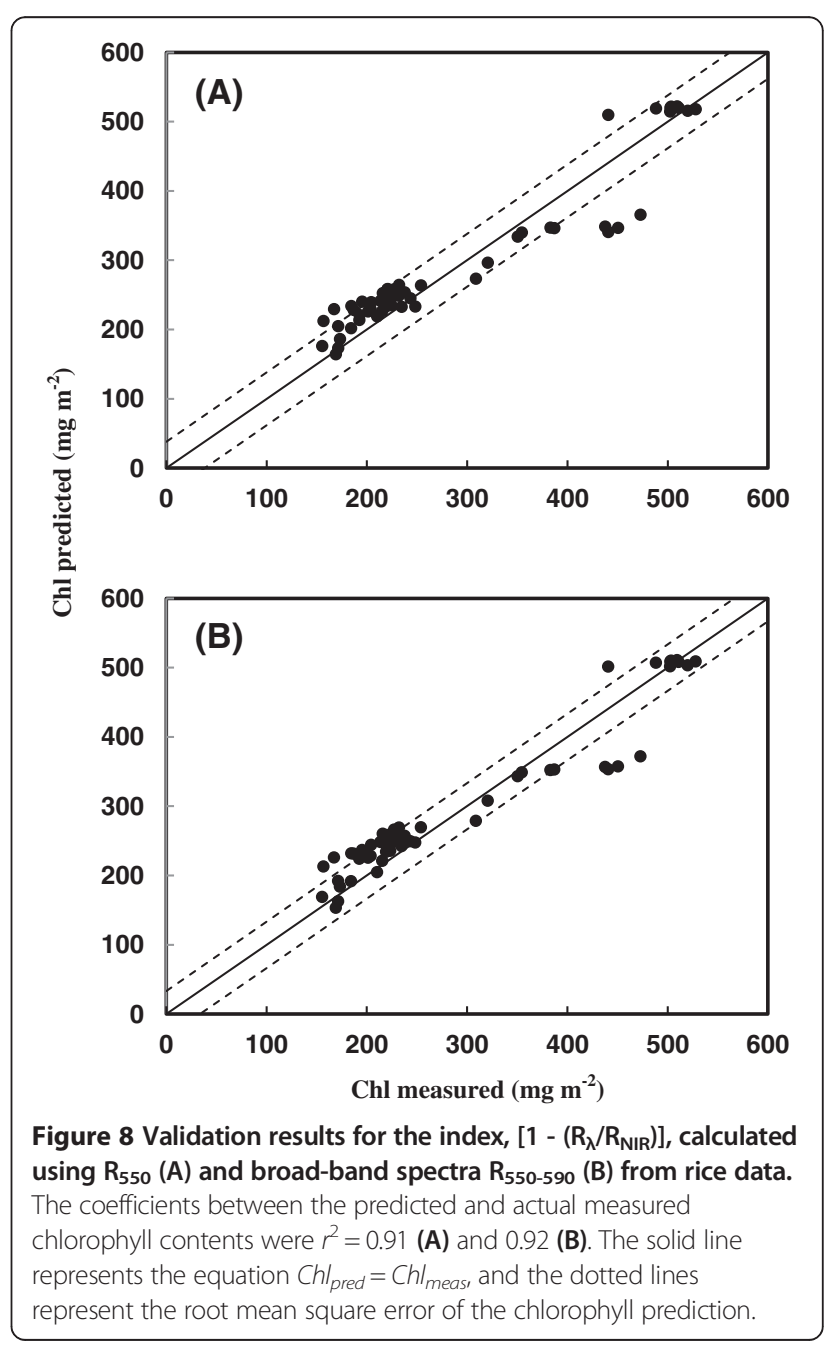


Furthermore, the new index is more comprehensively applicable to nondestructively estimate the chlorophyll contents in any kind of leaves and fruits with any level of anthocyanins.

\section{Additional file}

\section{Additional file 1: Multilingual abstracts in the six official working} languages of the United Nations.

\section{Competing interests}

The authors declare that they have no competing interests.

\section{Authors' contributions}

WDH, MHH, PYC, CMY conceived and designed the experiments. WDH, KHL $\mathrm{MHH}, \mathrm{MYH}, \mathrm{ZWY}$ performed the experiments. WDH, MHH, MYH, ZWY analyzed the data. $\mathrm{WDH}, \mathrm{MHH}, \mathrm{KHL}$ wrote the manuscript. All authors read and approved the final manuscript

\section{Acknowledgements}

We thank Mr. Chang-Chang Chen for help with the greenhouse work and laboratory experiments.

\section{Author details}

${ }^{1}$ Department of Agronomy, National Taiwan University, Daan, Taipei, ROC 106, Taiwan. ${ }^{2}$ Graduate Institute of Biotechnology, Chinese Culture University, Xulin, Taipei, ROC 111, Taiwan. ${ }^{3}$ Refining and Manufacturing Research Institute, CPC Corporation, Taiwan, Chiayi ROC 600, Taiwan. ${ }^{4}$ Biodiversity Research Center, Academia Sinica, Nangang, Taipei, ROC 115, Taiwan. ${ }^{5}$ Taoyuan District Agricultural Research and Extension Station, Council of Agriculture, Taoyuan, ROC 327, Taiwan. ${ }^{6}$ Department of Food, Health and Nutrition Science, Chinese Culture University, Xulin, Taipei, ROC 111, Taiwan.

Received: 24 October 2013 Accepted: 11 November 2013

Published: 30 January 2014

\section{References}

Blackburn GA (2007) Hyperspectral remote sensing of plant pigments. J Exp Bot 58:855-867

Buschmann C, Nagel E (1993) In vivo spectroscopy and internal optics of leaves as basis for remote sensing of vegetation. Int J Remote Sens 14:711-722

Chappelle EW, Kim MS, McMurtrey JE (1992) Ratio analysis of reflectance spectra (RARS): an algorithm for the remote estimation of the concentrations of chlorophyll a, chlorophyll b, and carotenoids in soybean leaves. Remote Sens Environ 39:239-247

Curran PJ, Dungan JL, Macler BA, Plummer SE (1991) The effect of a red leaf pigment on the relationship between red edge and chlorophyll concentration. Remote Sens Environ 35:69-76

Dawson TP, Curran PJ, Plummer SE (1998) LIBERTY- modeling the effects of leaf biochemical concentration on reflectance spectra. Remote Sens Environ 65:50-60

Dillen SY, Beeck MO, Hufkens K, Buonanduci M, Phillips NG (2012) Seasonal patterns of foliar reflectance in relation to photosynthetic capacity and color index in two co-occurring tree species, quercus rubra and betula papyrifera. Agric For Meteor 160:60-68

Filella I, Serrano I, Serra J, Peňuelas J (1995) Evaluating wheat nitrogen status with canopy reflectance indices and discriminant analysis. Crop Sci 35:1400-1450

Gamon JA, Surfus JS (1999) Assessing leaf pigment content and activity with a reflectometer. New Phytol 143:105-117

Gitelson AA, Merzlyak MN (1994) Spectral reflectance changes associated with autumn senescence of asculus hippocastamum and Acer platanoides leaves, Spectral features and relation to chlorophyll estimation. J Plant Physiol 143:286-292

Gitelson AA, Merzlyak MN (1996) Signature analysis of leaf reflectance spectral: algorithm development for remote sensing of chlorophyll. J Plant Physiol 148:494-500

Gitelson AA, Merzlyak MN (1997) Remote estimation of chlorophyll content in higher plant leaves. Int J Remote Sens 18:291-298
Gitelson AA, Merzlyak MN, Chivkunova OB (2001) Optical properties and nondestructive estimation of anthocyanin content in plant leaves. Photochem Photobiol 74:38-45

Gitelson AA, Zur Y, Chivkunova OB, Merzlyak MN (2002) Assessing carotenoid content in plant leaves with reflectance spectroscopy. Photochem Photobiol 75:272-281

Gitelson AA, Gritz Y, Merzlyak MN (2003) Relationships between leaf chlorophyll content and spectral reflectance and algorithms for non-destructive chlorophyll assessment in higher plant leaves. J Plant Physiol 160:271-282

Gitelson AA, Keydan GP, Merzlyak MN (2006) Three-band model for noninvasive estimation of chlorophyll, carotenoids, and anthocyanin contents in higher plant leaves. Geophys Res Lett 33:L11402. doi:10.1029/2006GL026457

Gould KS, Kuhn DN, Lee DW, Oberbauer SF (1995) Why leaves are sometimes red. Nature 378:241-242

Huang N, Niu Z, Zhan Y, Xu S, Tappert MC, Wu C, Huang W, Gao S, Hou X, Cai D (2012) Relationships between soil respiration and photosynthesis-related spectral vegetation indices in two cropland ecosystems. Agric For Meteor $160: 80-89$

Inoue Y, Morinaga S, Shibayama M (1993) Non-destructive estimation of water status of intact crop leaves based on spectral reflectance measurements. Jap J Crop Sci 62:462-469

Jacquemoud S, Ustin SL, Verdebout J, Schmuck G, Andreoli G, Hosgood B (1996) Estimating leaf biochemistry using the PROSPECT leaf optical properties model. Remote Sens Environ 56:194-202

Ji SB, Yokoi M, Saito N, Mao LS (1992) Distribution of anthocyanins in aceraceae leaves. Biochem Systematic Ecol 20:771-781

Lichtenthaler HK, Gitelson AA, Lang M (1996) Non-destructive determination of chlorophyll content of leaves of a green and an aurea mutant of tobacco by reflectance measurements. J Plant Physiol 148:483-493

Main R, Cho MA, Mathieu R, O'Kennedy MM, Ramoelo A, Koch S (2011) An investigation into robust spectral indices for leaf chlorophyll estimation. J Photogrammetry Remote Sens 66:751-761

Mancinelli AL, Huang-Yang CP, Lindquist P, Anderson O, Rabino I (1975) Photocontrol of anthocyanin synthesis, III. The action of streptomycin on the synthesis of chlorophyll and anthocyanin. Plant Physiol 55:251-257

Merzlyak MN, Gitelson AA (1995) Why and what for the leaves are yellow in autumn? On the interpretation of optical spectra of senescing leaves (Acer platanoides L.). J Plant Physiol 145:315-320

Merzlyak MN, Solovchenko AE, Gitelson AA (2003) Reflectance spectral features and non-destructive estimation of chlorophyll, carotenoid and anthocyanin content in apple fruit. Postharvest Biol Technol 27:197-211

Moran JA, Mitchell AK, Goodmanson G, Stockburger KA (2000) Differentiation among effects on nitrogen fertilization treatments on conifer seedlings by foliar reflectance: a comparison of methods. Tree Physiol 20:1113-1120

Porra RJ, Thompson WA, Kriedemann PE (1989) Determination of accurate extraction and simultaneously equation for assaying chlorophyll $\mathrm{a}$ and $\mathrm{b}$ extracted with different solvents: verification of the concentration of chlorophyll standards by atomic absorption spectroscopy. Biochem Biophys Acta 975:384-394

Zou X, Shi J, Hao L, Zhao J, Mao H, Chen Z, Li Y, Mel H (2011) In vivo noninvasive detection of chlorophyll distribution in cucumber (cucumis sativus) leaves by indices based on hyperspectral imaging. Anal Chim Acta 706:105-112

\section{doi:10.1186/1999-3110-55-11}

Cite this article as: Huang et al.: Eliminating interference by anthocyanin in chlorophyll estimation of sweet potato (Ipomoea batatas L.) leaves. Botanical Studies 2014 55:11. 